Working Paper 01-34

Statistics and Econometrics Series 22

July 2001
Departamento de Estadística y Econometría

Universidad Carlos III de Madrid

Calle Madrid, 126

28903 Getafe (Spain)

Fax (34) 91 624-98-49

\title{
FORECAST OF THE EXPECTED NON-EPIDEMIC MORBIDITY OF ACUTE DISEASES USING RESAMPLING METHODS
}

\author{
Andrés M. Alonso and Juan Romo*
}

\begin{abstract}
In epidemiological surveillance it is important that any unusual increase of reported cases be detected as rapidly as possible. Reliable forecasting based on a suitable time series model for an epidemiological indicator is necessary for estimating the expected non-epidemic indicator and to elaborate an alert threshold. Time series analysis of acute diseases often use Gaussian autoregressive integrated moving average models. However, these approaches could be adversely affected by departures from the true underlying distribution. The objective of this paper is to introduce a bootstrap procedure for obtaining prediction intervals in linear models in order to avoid the normality assumption. We present a Monte Carlo study comparing the finite sample properties of the bootstrap prediction intervals with those of alternative methods. Finally, we illustrate the performance of the proposed method with a meningococcal disease incidence series.
\end{abstract}

Keywords: sieve bootstrap; prediction intervals; time series; linear processes.

* Dept. of Statistics and Econometrics, Univ. Carlos III de Madrid, Spain. Alonso, email: alonsoam@est-econ.uc3m.es; Romo: romo@est-econ.uc3m.es; This work was partially supported by the I+D+I project BEC 2000-0167 and Cátedra de Calidad BBVA. AMS (1990) Subject Classifications: 62M10, 62M20, 62G09. 


\section{Introduction}

Epidemiological surveillance is an important component for the public health system because it provides useful information about the course of diseases and other health events that may lead to corrective actions from the health sector. Epidemiological surveillance consists of three interrelated components: continuous systematic data collection, use of models to make inference from data, and rapid dissemination of findings to help the public health decisionmaking process $[1,2]$.

One important task for surveillance systems is accurate forecasting of the case occurrence of health events and detection of abnormal values in case occurrence. A typical approach used to investigate this problem is based on using time series models for specific health variables $[2,3,4,5,6,8]$. In particular, the seasonal autoregressive integrated moving average models ARIMA (also known as Box-Jenkins models [7]) are used with success in different situations $[3,4,5,6,8]$.

The Box-Jenkins approach assume that series $\left\{X_{t}\right\}_{t \in \mathbb{Z}}$ follows a linear finite dimensional model with a known error distribution. Usually it assumes a Gaussian seasonal autoregressivemoving average model $\operatorname{ARIMA}(p, d, q)(P, D, Q)_{s}$ :

$$
\boldsymbol{\phi}(B) \boldsymbol{\Phi}(B)(1-B)^{d}\left(1-B^{s}\right)^{D} X_{t}=\boldsymbol{\theta}(B) \boldsymbol{\Theta}(B) \varepsilon_{t},
$$

where $\phi(B)=1-\phi_{1} B-\cdots-\phi_{p} B^{p}$ and $\boldsymbol{\Phi}(B)=1-\Phi_{1} B^{s}-\cdots-\Phi_{P} B^{s P}$ are the regular and seasonal autoregressive polynomials, $\boldsymbol{\theta}(B)=1-\theta_{1} B-\cdots-\theta_{q} B^{q}$ and $\boldsymbol{\Theta}(B)=1-\Theta_{1} B^{s}-$ $\cdots-\Theta_{Q} B^{s Q}$ are the regular and seasonal moving average polynomials, $B$ is the backward shift operator such that $B X_{t}=X_{t-1}$ and $B^{s} X_{t}=X_{t-s}$, and the variables $\left\{\varepsilon_{t}\right\}_{t \in \mathbb{Z}}$ are independent and identically distributed random variables with $\varepsilon \sim \mathcal{N}\left(0, \sigma_{\varepsilon}^{2}\right)$. In such a case, if the orders are known, a maximum likelihood procedure could be employed for estimating the parameters. The maximum likelihood estimates could then be plugged into the prediction intervals:

$$
\widehat{\mathrm{E}}\left[X_{T+h} \mid \boldsymbol{X}_{1}^{T}\right] \pm z_{\alpha / 2}\left(\widehat{\sigma}_{\varepsilon}^{2} \sum_{j=0}^{h-1} \widehat{\psi}_{j}^{2}\right)^{1 / 2}
$$

where $\widehat{\mathrm{E}}\left[X_{T+h} \mid \boldsymbol{X}_{1}^{T}\right]$ is the linear predictor obtained using model (1), and the $\widehat{\psi}_{j}$ are the estimated coefficients of the moving average representation (see Section 9.2 in [7]).

However, these prediction intervals could be adversely affected by departures from the true underlying distribution. Using a Monte Carlo study, it was shown that the standard (Gaussian) Box Jenkins method performs poorly given a skewed error distribution [9]. Also notice that prediction intervals (2) do not incorporate the uncertainty due to parameter estimation. The effects of parameter estimation are particularly important for small sample sizes and when the error distribution is not Gaussian [10].

A bootstrap method [9] has been proposed for estimating prediction intervals of an $\operatorname{AR}(p)$ process when $p$ is known. This method uses first a backward and then forward bootstrap that makes the procedure computationally expensive. Cao et al. [11] study an alternative conditional bootstrap method, which is computationally much faster. This conditional bootstrap has been generalized to $\operatorname{ARIMA}(p, d, q)$ processes with known $p, d$ and $q[10]$ and it also includes the parameter estimation variability. But, those bootstrap proposals assume that the 
orders of regular and seasonal polynomials are known. Alonso et al. [12] show that the $\operatorname{AR}(\infty)$-sieve bootstrap provides consistent prediction intervals for a general class of linear models that includes stationary and invertible ARMA processes. This sieve bootstrap has a nice nonparametric property, being model-free within the considered class of linear processes. Thus, the proposed bootstrap prediction intervals could be applied to a more general class of linear models without specifying a finite dimensional model as in previous bootstrap proposals.

In this paper, we extend the sieve bootstrap prediction method [12] to linear processes that admit the following $\operatorname{AR}(\infty)$ representation:

$$
\sum_{j=0}^{+\infty} \phi_{j} B^{j} \sum_{j=0}^{+\infty} \Phi_{j} B^{s j} Y_{t}=\varepsilon_{t}
$$

where $Y_{t}=(1-B)^{d}\left(1-B^{s}\right)^{D} X_{t}$, and $d, D$ and $s$ are known parameters, and the coefficients $\left\{\phi_{j}\right\}_{j=0}^{+\infty}$ and $\left\{\Phi_{j}\right\}_{j=0}^{+\infty}$ satisfy, for some $r>2$, that $\sum_{j=0}^{+\infty} j^{r}\left|\phi_{j}\right|<\infty$ and $\sum_{j=0}^{+\infty} j^{r}\left|\Phi_{j}\right|<\infty$, respectively. Notice that the above conditions are satisfied by models (1) since they have an exponential decay of $\left\{\phi_{j}\right\}_{j=0}^{+\infty}$ and $\left\{\Phi_{j}\right\}_{j=0}^{+\infty}$ in their $\operatorname{AR}(\infty)$ representation.

The remaining of this paper is organized as follows. Section 2 introduces the sieve bootstrap for estimating forecast intervals. Section 3 presents a Monte Carlo study comparing the finite sample properties of the sieve bootstrap with the Gaussian Box-Jenkins approach. Finally, in Section 4 the performance of the proposed method is illustrated with a real data example.

\section{Sieve bootstrap prediction intervals}

Let $\left\{X_{t}\right\}_{t \in \mathbb{Z}}$ be a real valued process that admits an $\operatorname{AR}(\infty)$ representation as in (3). The method proceeds as follows:

1. Given a sample $\left(X_{1}, X_{2}, \ldots, X_{T}\right)$, obtain the differentiated series $Y_{t}=(1-B)^{d}(1-$ $\left.B^{s}\right)^{D} X_{t}$ with $t=d+s D+1, d+s D+2, \ldots, T$.

2. Given $\left(Y_{d+s D+1}, Y_{d+s D+2}, \ldots, Y_{T}\right)$ select the order $p=p(T)$ and $P=P(T)$ of the regular and seasonal autoregressive approximation by the AICC criterion: AICC $=$ $-T \log \left(\sigma^{2}\right)+2(p+P+1) T /(T-p-P-2)$ with $0 \leq p \leq p_{\max }$ and $0 \leq P \leq P_{\max }$.

The AICC criterion [14] is a bias-corrected version of AIC and it has a more extreme penalty for large-order models which counteracts the overfitting nature of AIC. Also, the AICC is less affected than AIC or BIC by changes in the value of the maximum order $p_{\max }$ considered [14].

3. Construct the least square estimators of the autoregressive coefficients: $\widehat{\phi}_{p}=\left(\widehat{\phi}_{1}, \widehat{\phi}_{2}, \ldots, \widehat{\phi}_{p}\right)^{\prime}$ and $\widehat{\boldsymbol{\Phi}}_{P}=\left(\widehat{\Phi}_{1}, \widehat{\Phi}_{2}, \ldots, \widehat{\Phi}_{P}\right)^{\prime}$, i.e. $\widehat{\boldsymbol{\phi}}_{p}$ and $\widehat{\boldsymbol{\Phi}}_{P}$ are the solutions of:

$$
\min _{\boldsymbol{\phi}_{p}, \mathbf{\Phi}_{P}}\left\{\sum_{t=d+s(D+P)+p+1}^{T}\left(\sum_{j=0}^{p} \phi_{j} B^{j} \sum_{j=0}^{P} \Phi_{j} B^{s j} Y_{t}\right)^{2}\right\} .
$$


4. Compute the residuals:

$$
\widehat{\varepsilon}_{t}=\sum_{j=0}^{p} \widehat{\phi}_{j} B^{j} \sum_{j=0}^{P} \widehat{\Phi}_{j} B^{s j} Y_{t} ; \widehat{\phi}_{0}=1, \widehat{\Phi}_{0}=1, t \in(d+s(D+P)+p+1, \ldots, T) .
$$

5. Define the empirical distribution function of the centred residuals:

$$
\widehat{F}_{\widetilde{\varepsilon}}(x)=(T-(d+s(D+P)+p))^{-1} \sum_{t=d+s(D+P)+p+1}^{T} I\left\{\widetilde{\varepsilon}_{t} \leq x\right\},
$$

where $\tilde{\varepsilon}_{t}=\widehat{\varepsilon}_{t}-\widehat{\varepsilon}^{(\cdot)}$ and $\widehat{\varepsilon}^{(\cdot)}=(T-(d+s(D+P)+p))^{-1} \sum_{t=d+s(D+P)+p+1}^{T} \widehat{\varepsilon}_{t}$.

6. Draw a resample $\varepsilon_{t}^{*}$ of i.i.d. observations from $\widehat{F}_{\widetilde{\varepsilon}}$.

7. Define $X_{t}^{*}$ by the recursion:

$$
\sum_{j=0}^{p} \widehat{\phi}_{j} B^{j} \sum_{j=0}^{P} \widehat{\Phi}_{j} B^{s j}(1-B)^{d}\left(1-B^{s}\right)^{D} X_{t}^{*}=\varepsilon_{t}^{*},
$$

where the starting $d+s(D+P)+p$ observations $X_{1}^{*}, X_{2}^{*}, \ldots, X_{d+s(D+P)+p}^{*}$ are drawn with equal probability from all the $T-(d+s(D+P)+p+1)$ possible blocks of consecutive observations of the original series.

In practice we generate an $\operatorname{ARIMA}(p, d, 0)(P, D, 0)_{s}$ resample using (6) with sample size equal to $T+100+10 s$ and then discard the first $100+10 s$ observations in order to minimize the effect of starting values.

In the next step we estimate the parameters of the bootstrap series generated in step 6. This allows us to introduce the parameter estimation variability on estimated prediction intervals.

8. Given $\left(X_{1}^{*}, \ldots, X_{T}^{*}\right)$ from the previous step, obtain the bootstrap differentiated series $\left(Y_{d+s D+1}^{*}, Y_{d+s D+2}^{*}, \ldots, Y_{T}^{*}\right)$ and compute the estimation of the autoregressive coefficients $\widehat{\boldsymbol{\phi}}_{p}^{*}=\left(\widehat{\phi}_{1}^{*}, \widehat{\phi}_{2}^{*}, \ldots, \widehat{\phi}_{p}^{*}\right)^{\prime}$ and $\widehat{\boldsymbol{\Phi}}_{P}^{*}=\left(\widehat{\Phi}_{1}^{*}, \widehat{\Phi}_{2}^{*}, \ldots, \widehat{\Phi}_{P}^{*}\right)^{\prime}$, as in step 3 .

Steps 1 to 8 are not effective for bootstrap prediction, because the algorithm does not replicate the conditional distribution of $X_{T+h}$ given the observed data. But, if we fix the last $p+d+s(P+D)$ observations, we can obtain resamples of the future values $X_{T+h}^{*}$ given $X_{T-(p+d+(P+D) s)+1}^{*}=X_{T-(p+d+(P+D) s)+1}, \ldots, X_{T}^{*}=X_{T}$.

9. Compute future bootstrap observations by the recursion:

$$
\sum_{j=0}^{p} \widehat{\phi}_{j}^{*} B^{j} \sum_{j=0}^{P} \widehat{\Phi}_{j}^{*} B^{s j}(1-B)^{d}\left(1-B^{s}\right)^{D} X_{t}^{*}=\varepsilon_{t}^{*} ; \text { for } t=T+1, T+2, \ldots, T+h,
$$

where $h>0$, and $X_{t}^{*}=X_{t}$, for $t \leq T$. 
As an illustration of the above step, we consider that the selected model in step 1-2 is an $\operatorname{ARIMA}(1,1,0)(1,1,0)_{12}$ :

$$
(1-\phi B)\left(1-\Phi B^{12}\right)(1-B)\left(1-B^{12}\right) X_{t}=\varepsilon_{t} .
$$

Once the parameters $\phi$ and $\Phi$ of model (8) have been estimated and the bootstrap replicate of the series has been constructed by (6), we obtain the bootstrap estimates $\widehat{\phi}^{*}$ and $\widehat{\Phi}^{*}$. Then, using (7), we generate the future bootstrap observations as follows:

$$
\begin{array}{rcc}
X_{T+1}^{*}= & \left(1+\widehat{\phi}^{*}\right) X_{T}-\widehat{\phi}^{*} X_{T-1}+\left(1+\widehat{\Phi}^{*}\right) X_{T-11}-\left(1+\widehat{\phi}^{*}+\widehat{\Phi}^{*}+\widehat{\phi}^{*} \widehat{\Phi}^{*}\right) X_{T-12}+ \\
& +\left(\widehat{\phi}^{*}+\widehat{\phi}^{*} \widehat{\Phi}^{*}\right) X_{T-13}-\widehat{\Phi}^{*} X_{T-23}+\left(\widehat{\Phi}^{*}+\widehat{\phi}^{*} \widehat{\Phi}^{*}\right) X_{T-24}-\widehat{\phi}^{*} \widehat{\Phi}^{*} X_{T-25}+\varepsilon_{T+1}^{*} \\
X_{T+2}^{*}= & \left(1+\widehat{\phi}^{*}\right) X_{T+1}^{*}-\widehat{\phi}^{*} X_{T}+\left(1+\widehat{\Phi}^{*}\right) X_{T-10}-\left(1+\widehat{\phi}^{*}+\widehat{\Phi}^{*}+\widehat{\phi}^{*} \widehat{\Phi}^{*}\right) X_{T-11}+ \\
& +\left(\widehat{\phi}^{*}+\widehat{\phi}^{*} \widehat{\Phi}^{*}\right) X_{T-12}-\widehat{\Phi}^{*} X_{T-22}+\left(\widehat{\Phi}^{*}+\widehat{\phi}^{*} \widehat{\Phi}^{*}\right) X_{T-23}-\widehat{\phi}^{*} \widehat{\Phi}^{*} X_{T-24}+\varepsilon_{T+2}^{*} \\
\vdots & \vdots & \vdots \\
X_{T+h}^{*}= & \left(1+\widehat{\phi}^{*}\right) X_{T+h-1}^{*}-\widehat{\phi}^{*} X_{T+h-2}^{*}+\left(1+\widehat{\Phi}^{*}\right) X_{T+h-12}^{*}-\left(1+\widehat{\phi}^{*}+\widehat{\Phi}^{*}+\widehat{\phi}^{*} \widehat{\Phi}^{*}\right) X_{T+h-13}^{*}+ \\
& +\left(\widehat{\phi}^{*}+\widehat{\phi}^{*} \widehat{\Phi}^{*}\right) X_{T+h-14}^{*}-\widehat{\Phi}^{*} X_{T+h-24}^{*}+\left(\widehat{\Phi}^{*}+\widehat{\phi}^{*} \widehat{\Phi}^{*}\right) X_{T+h-25}^{*}-\widehat{\phi}^{*} \widehat{\Phi}^{*} X_{T+h-26}^{*}+\varepsilon_{T+h}^{*} .
\end{array}
$$

Notice that, in the recursions above, $\left(X_{T}, X_{T-1}, \ldots, X_{T-25}\right)$ are kept fixed in the different bootstrap replicates of $\left(X_{T+1}^{*}, X_{T+2}^{*}, \ldots, X_{T+h}^{*}\right)$, while $\left(\varepsilon_{T+1}^{*}, \varepsilon_{T+2}^{*}, \ldots, \varepsilon_{T+h}^{*}\right)$ changes from one replication to another.

Finally, the bootstrap distribution $F_{X_{T+h}^{*}}^{*}$ of $X_{T+h}^{*}$ is used to approximate the unknown distribution of $X_{T+h}$ given the observed sample. A Monte Carlo estimate $\widehat{F}_{X_{T+h}^{*}}^{*}$ is obtained by repeating the steps 6 to $9 B$ times. The estimated $(1-\alpha) \%$ prediction interval for $X_{T+h}$ is given by $\left[Q^{*}(\alpha / 2), Q^{*}(1-\alpha / 2)\right]$, where $Q^{*}(\cdot)=\widehat{F}_{X_{T+h}^{*-1}}^{*}(\cdot)$ are the quantiles of the estimated bootstrap distribution.

\section{Simulation study}

In this section, we present the results of several Monte Carlo studies comparing the sieve bootstrap approach (S) with the standard Gaussian forecast intervals (BJ). The Monte Carlo studies include four models based on actual epidemiological surveillance data $[16,5,6,17]$. First, we consider a stationary $\mathrm{AR}(5)$ process without seasonal pattern. Then, we compare the two prediction methods in multiplicative seasonal ARIMA processes for quarterly, monthly and weekly data.

Model 1: Autoregressive model fitted by Cardinal et al. [16] to meningoccocal infection series for period 1986-1993:

$$
X_{t}-0.010 X_{t-1}+0.113 X_{t-2}-0.024 X_{t-3}-0.020 X_{t-4}-0.241 X_{t-5}=\varepsilon_{t},
$$

where $\mathrm{E}\left[\varepsilon_{t}\right]=0$ and $\mathrm{E}\left[\varepsilon_{t}^{2}\right]=\sigma_{\varepsilon}^{2}=1.948$. 
Model 2: Seasonal ARIMA model fitted by Zaidi et al. [5] to primary and secondary syphilis cases $\left(Z_{t}\right)$ in women for period 1966-1987 (quarterly data):

$$
(1-B)\left(1-B^{4}\right) X_{t}=\left(1-0.755 B^{4}\right) \varepsilon_{t},
$$

where $X_{t}=\log \left(Z_{t}\right), \mathrm{E}\left[\varepsilon_{t}\right]=0$ and $\mathrm{E}\left[\varepsilon_{t}^{2}\right]=\sigma_{\varepsilon}^{2}=0.004$.

Model 3: Seasonal ARIMA model fitted by Watier et al. [6] to Salmonella infections report series $\left(Z_{t}\right)$ for period 1978-1988 (monthly data):

$$
(1-0.347 B)\left(1-B^{12}\right) X_{t}=\left(1-0.707 B^{12}\right) \varepsilon_{t},
$$

where $\mathrm{E}\left[\varepsilon_{t}\right]=0$ and $\mathrm{E}\left[\varepsilon_{t}^{2}\right]=\sigma_{\varepsilon}^{2}=118.81$.

Model 4: Seasonal ARIMA model fitted by Williamson and Hudson [17] to hepatitis type A report series $\left(Z_{t}\right)$ for period 1980-1988 (weekly data):

$$
\left(1-0.22 B^{52}\right)(1-B)\left(1-B^{52}\right) X_{t}=(1-0.90 B)\left(1-0.82 B^{52}\right) \varepsilon_{t},
$$

where $X_{t}=\sqrt{Z_{t}}, \mathrm{E}\left[\varepsilon_{t}\right]=0$ and $\mathrm{E}\left[\varepsilon_{t}^{2}\right]=\sigma_{\varepsilon}^{2}=2.948$.

For each model considered, we generate series with several choices of error distribution, in particular, Gaussian $\mathcal{N}\left(0, \sigma_{\varepsilon}^{2}\right)$, exponential $\mathcal{E} x p\left(\sigma_{\varepsilon}\right)$, and reversed exponential $-\mathcal{E} x p\left(\sigma_{\varepsilon}\right)$. In all cases, we have centered the errors to have zero mean and variance equal to $\mathrm{E}\left[\varepsilon_{t}^{2}\right]$.

To compare the different prediction intervals, we use their mean coverage and length, and the proportions of observations lying out to the left and to the right of the interval. These quantities are estimated as follows:

1. For a combination of model, sample size and error distribution, simulate a series, and generate $R=1000$ future values $X_{T+h}$, where lead times are $h=1,2$, and 3 .

2. For each procedure obtain the $(1-\alpha) \%$ prediction interval $\left[Q_{M}(\alpha / 2), Q_{M}(1-\alpha / 2)\right]$ based on $B=1000$ bootstrap resamples for $\mathrm{M}=\mathrm{S}$, and based on Gaussian distribution for $\mathrm{M}=\mathrm{BJ}$.

3. The coverage for each method is estimated as $C_{M}=\#\left\{Q_{M}(\alpha / 2) \leq X_{T+h}^{r} \leq Q_{M}(1-\right.$ $\alpha / 2)\} / R$, where $X_{T+h}^{r}$ with $r=1, \ldots, R$, being the $R$ future values generated in the first step.

In steps 1 and 2 we obtain the "theoretical" and estimated interval lengths using $L_{T}=$ $X_{T+h}^{\lceil R(1-\alpha / 2)\rceil}-X_{T+h}^{\lceil R \alpha / 2\rceil}$, and $L_{M}=Q_{M}(1-\alpha / 2)-Q_{M}(\alpha / 2)$. Finally, steps 1 to 3 are repeated $S=200$ times to obtain $C_{M, i}, L_{M, i}$ with $i=1, \ldots, S$, and we calculate the estimates:

$$
\begin{array}{ll}
\bar{C}_{M} & =S^{-1} \sum C_{M, i} \\
S E\left(\bar{C}_{M}\right) & =\left(S^{-1}(S-1)^{-1} \sum\left(C_{M, i}-\bar{C}_{M}\right)^{2}\right)^{1 / 2} \\
\bar{L}_{M} & =S^{-1} \sum L_{M, i} \\
S E\left(\bar{L}_{M}\right) & =\left(S^{-1}(S-1)^{-1} \sum\left(L_{M, i}-\bar{L}_{M}\right)^{2}\right)^{1 / 2}
\end{array}
$$


where $\bar{L}_{T}=S^{-1} \sum L_{T, i}$ is the estimated "true" mean interval length, and $\bar{C}_{T}=(1-\alpha) \%$ is the nominal coverage.

The results for Model 1 are presented in Tables 1-3, using two sample sizes $n=70$ and 130 and the three error distributions, nominal coverage 95\%, and lead times $h=1,2$ and 3. Comparing BJ and S intervals for the Gaussian innovation distribution (Table 1) the BJ method obtains coverage closer to the nominal values. Notice that BJ intervals are built assuming the correct error distribution and also assuming the true generating model.

Table 2 (3) reports the Monte Carlo results for the same model but with innovations generated by a positively (negatively) skewed distribution. It is observed that the BJ intervals exhibit an asymmetric proportions of under and over coverage. In Figure 1, we present the empirical, BJ and S densities estimates for one-step ahead predictions of one series generated by Model 1 with exponential innovations and sample size $n=130$. It can be seen that the BJ method is not able to handle the asymmetry of the error distribution.

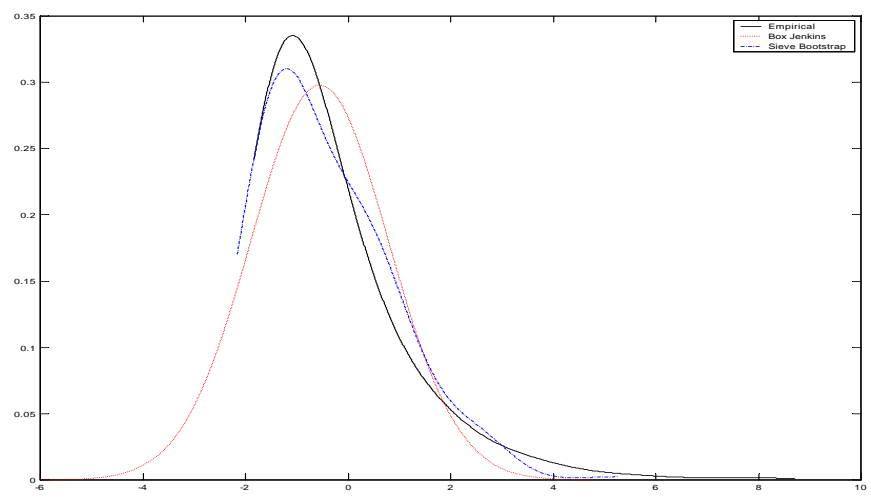

Figure 1: Kernel densities estimates of one-step ahead predictions of one series generated by Model 1.

The results for Models 2, 3 and 4 are presented in Tables 4-6, 7-9 and 10-12, using two sample sizes $n=80$ and 160, $n=120$ and 240, and $n=260$ and 520, respectively. In all cases we consider the three error distributions, nominal coverage $95 \%$, and lead times $h=1,2$ and 3. As in Model 1, sieve bootstrap method perform reasonably well. Notice that in these cases, the sieve approach never uses the correct model since the generating models include some MA components.

\section{Real data example}

In this section, we study the meningococcal disease incidence time series from MontréalCentre region during the period January 1986 - December 1993. This incidence time series was previously modeled by a Gaussian AR(5) model and by an integer-valued autoregressive model [16]. For the sake of comparison, we will use $p(T)=5$ in sieve bootstrap prediction 
intervals.

In Figure 2, we present the one-step ahead prediction intervals for the last 13 available observations as in [16]. An upward shift of the sieve bootstrap limits can be observed, revealing the asymmetric distribution of the residuals.

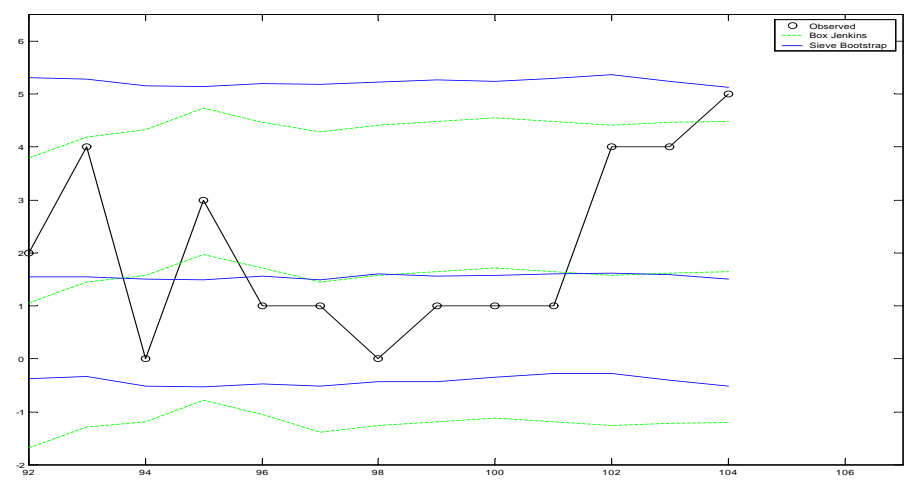

Figure 2: Forecasts and 95\% one-step ahead forecast interval limits for meningococcal infection incidence during the year 1993.

A kernel density estimate for one-step ahead sieve bootstrap prediction at time $T=92$ together with the corresponding Gaussian prediction density is presented in Figure 3. It is clear that density estimate obtained using the sieve bootstrap procedure captures the asymmetry observed in the residual distribution. In [16], it was shown that the residuals from the realvalued autoregressive model exhibit positive asymmetry (see their Figures 1(d) and 1(e)).

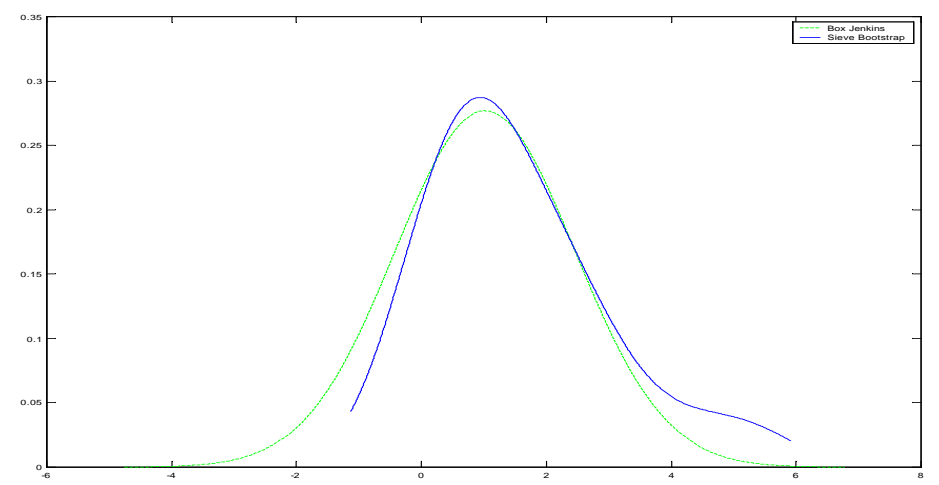

Figure 3: Kernel density estimate of one-step ahead predictions for meningococcal incidence series. 


\section{Acknowledgements}

The authors are grateful to Prof. J. Lambert and R. Roy for providing the data. We would like to thank Mike Wiper for carefully reading that greatly improved the paper. This work was partially supported by the Dirección General de Investigación Científica y Técnica of Spain and Cátedra de Calidad BBVA.

\section{Conclusions}

In this paper we have proposed a bootstrap method to estimate prediction density and prediction intervals for a general class of linear models including seasonal $\operatorname{ARIMA}(p, d, q)(P, D, Q)_{s}$ models. This prediction resampling procedure does not require us to specify a finite dimensional models as in previous bootstrap and standard Gaussian approaches. As in [9] and [10], we have illustrated that Box-Jenkins intervals are adversely affected by departures from normality assumption. Monte Carlo simulations have shown that the proposed method performs well given normality and given asymmetric distributions.

\section{References}

[1] Thacker SB, Berkelman RL. Public Health Surveillance in the United States. Epidemiologic Reviews 1988; 10: 164-190.

[2] Serfling RE. Methods for Current Statistical Analysis of Excess Pneumonia-Influenza Deaths. Public Health Reports 1963; 78: 494-50.

[3] Choi K, Thacker SB. An Evaluation of Influenza Mortality Surveillance 1962-1979. I Time Series Forecast of Expected Pneumonia and Influenza Deaths. American Journal of Epidemiology 1981; 113: 215-226.

[4] Helfentein U. Box-Jenkins Modelling of some Viral Infectious Diseases. Statistics in Medicine 1986; 5: 37-47.

[5] Zaidi AA, Schnell DJ, Reynolds GH. Time Series Analysis of Syphilis Surveillance Data. Statistics in Medicine 1989; 8: 353-362.

[6] Watier L, Richarson S, Hubert B. A Time Series Construction of an Alert Threshold with Application to S. Bovimorbificans in France. Statistics in Medicine 1991; 10: 1493-1509.

[7] Box GEP, Jenkins GM. Time Series Analysis Forecasting and Control (1st edn), HoldenDay: Oakland, 1976.

[8] Aguirre A, González E. Forecast of Acute Respiratory Infections: Expected Nonepidemic Morbidity in Cuba. Memorias do Instituto Oswaldo Cruz 1992; 87: 433-436.

[9] Thombs LA, Schucany WR. Bootstrap prediction intervals for autoregression. Journal of the American Statistical Association 1990; 85: 486-492. 
[10] Pascual, L, Romo J, Ruiz E. Effects of parameter estimation on prediction densities: a bootstrap approach. International Journal of Forecasting 2001; 17: 83-103.

[11] Cao R, Febrero-Bande M, González-Manteiga W, Prada-Sánchez JM, García-Jurado I. Saving computer time in constructing consistent bootstrap prediction intervals for autoregressive processes. Communications in Statistics. Simulation and Computation 1997; 26: $961-978$.

[12] Alonso AM, Peña D, Romo J. Forecasting time series with sieve bootstrap. Journal of Statistical Planning and Inference 2001; in press.

[13] Bühlmann, P. Sieve bootstrap for time series. Bernoulli 1997; 8: 123-148.

[14] Hurvich CM, Tsai C-L. Regression and time series model selection in small samples. Biometrika 1989; 76: 297-307.

[15] Bhansali RJ. A simulation study of autoregressive and window estimators of the inverse autocorrelation function. Journal of the Royal Statistical Society. Series C 1983; 32: 141-149.

[16] Cardinal M, Roy R, Lambert J. On the application of integer-valued time series models for the analysis of diseases incidence. Statistics in Medicine 1999; 18: 2025-2039.

[17] Williamson GD, Hudson GW. A monitoring system for detecting aberrations in public health surveillance reports. Statistics in Medicine 1999; 18: 3283-3298. 
Table 1: Simulation results for Model 1, with Gaussian residuals.

\begin{tabular}{|c|c|c|c|c|c|}
\hline Lag & Sample size & Method & $C_{M}(\mathrm{se})$ & Cov. (i./s.) & $L_{M}(\mathrm{se})$ \\
\hline $\mathrm{h}$ & $\mathrm{n}$ & Theoretical & $95 \%$ & $2.50 \% / 2.50 \%$ & 5.47 \\
\hline 1 & 70 & $\mathrm{BJ}$ & $93.23(0.12)$ & $3.39 / 3.38$ & $5.45(0.02)$ \\
\hline & & $\mathrm{S}$ & $91.78(0.16)$ & $4.07 / 4.15$ & $5.37(0.02)$ \\
\hline $\mathrm{h}$ & $\mathrm{n}$ & Theoretical & $95 \%$ & $2.50 \% / 2.50 \%$ & 5.48 \\
\hline 2 & 70 & BJ & $93.71(0.10)$ & $3.11 / 3.18$ & $5.50(0.02)$ \\
\hline & & $\mathrm{S}$ & $92.36(0.13)$ & $3.75 / 3.88$ & $5.40(0.02)$ \\
\hline $\mathrm{h}$ & $\mathrm{n}$ & Theoretical & $95 \%$ & $2.50 \% / 2.50 \%$ & 5.50 \\
\hline 3 & 70 & BJ & $94.15(0.10)$ & $2.87 / 2.98$ & $5.59(0.02)$ \\
\hline & & $\mathrm{S}$ & $92.85(0.12)$ & $3.42 / 3.73$ & $5.47(0.02)$ \\
\hline $\mathrm{h}$ & $\mathrm{n}$ & Theoretical & $95 \%$ & $2.50 \% / 2.50 \%$ & 5.47 \\
\hline 1 & 130 & BJ & $93.93(0.07)$ & $3.02 / 3.05$ & $5.43(0.01)$ \\
\hline & & $\mathrm{S}$ & $92.69(0.11)$ & $3.71 / 3.60$ & $5.33(0.02)$ \\
\hline $\mathrm{h}$ & $\mathrm{n}$ & Theoretical & $95 \%$ & $2.50 \% / 2.50 \%$ & 5.48 \\
\hline 2 & 130 & BJ & $94.16(0.07)$ & $2.86 / 2.98$ & $5.45(0.01)$ \\
\hline & & S & $92.98(0.10)$ & $3.43 / 3.59$ & $5.34(0.02)$ \\
\hline $\mathrm{h}$ & $\mathrm{n}$ & Theoretical & $95 \%$ & $2.50 \% / 2.50 \%$ & 5.50 \\
\hline 3 & 130 & BJ & $94.35(0.06)$ & $2.74 / 2.92$ & $5.51(0.01)$ \\
\hline & & S & $93.26(0.10)$ & $3.27 / 3.47$ & $5.40(0.01)$ \\
\hline
\end{tabular}

Table 2: Simulation results for Model 1, with exponential residuals.

\begin{tabular}{|c|c|c|c|c|c|}
\hline Lag & Sample size & Method & $C_{M}(\mathrm{se})$ & Cov. (i./s.) & $L_{M}(\mathrm{se})$ \\
\hline $\mathrm{h}$ & $\mathrm{n}$ & Theoretical & $95 \%$ & $2.50 \% / 2.50 \%$ & 5.09 \\
\hline 1 & 70 & $\mathrm{BJ}$ & $93.63(0.14)$ & $0.29 / 6.08$ & $5.42(0.03)$ \\
\hline & & $\mathrm{S}$ & $91.51(0.33)$ & $4.72 / 3.77$ & $5.28(0.04)$ \\
\hline $\mathrm{h}$ & $\mathrm{n}$ & Theoretical & $95 \%$ & $2.50 \% / 2.50 \%$ & 5.08 \\
\hline 2 & 70 & BJ & $94.00(0.11)$ & $0.13 / 5.87$ & $5.47(0.03)$ \\
\hline & & $\mathrm{S}$ & $92.96(0.27)$ & $3.37 / 3.67$ & $5.32(0.04)$ \\
\hline $\mathrm{h}$ & $\mathrm{n}$ & Theoretical & $95 \%$ & $2.50 \% / 2.50 \%$ & 5.20 \\
\hline 3 & 70 & BJ & $94.11(0.13)$ & $0.18 / 5.71$ & $5.55(0.03)$ \\
\hline & & $\mathrm{S}$ & $93.72(0.23)$ & $2.81 / 3.46$ & $5.43(0.04)$ \\
\hline $\mathrm{h}$ & $\mathrm{n}$ & Theoretical & $95 \%$ & $2.50 \% / 2.50 \%$ & 5.09 \\
\hline 1 & 130 & BJ & $94.34(0.07)$ & $0.03 / 5.63$ & $5.44(0.02)$ \\
\hline & & $\mathrm{S}$ & $92.29(0.28)$ & $4.47 / 3.25$ & $5.21(0.03)$ \\
\hline $\mathrm{h}$ & $\mathrm{n}$ & Theoretical & $95 \%$ & $2.50 \% / 2.50 \%$ & 5.08 \\
\hline 2 & 130 & BJ & $94.40(0.07)$ & $0.04 / 5.56$ & $5.46(0.02)$ \\
\hline & & S & $93.84(0.23)$ & $2.91 / 3.25$ & $5.23(0.03)$ \\
\hline $\mathrm{h}$ & $\mathrm{n}$ & Theoretical & $95 \%$ & $2.50 \% / 2.50 \%$ & 5.20 \\
\hline 3 & 130 & BJ & $94.49(0.06)$ & $0.01 / 5.50$ & $5.53(0.02)$ \\
\hline & & S & $94.35(0.17)$ & $2.44 / 3.22$ & $5.33(0.03)$ \\
\hline
\end{tabular}


Table 3: Simulation results for Model 1, with -exponential residuals.

\begin{tabular}{|c|c|c|c|c|c|}
\hline Lag & Sample size & Method & $C_{M}(\mathrm{se})$ & Cov. (i./s.) & $L_{M}(\mathrm{se})$ \\
\hline $\mathrm{h}$ & $\mathrm{n}$ & Theoretical & $95 \%$ & $2.50 \% / 2.50 \%$ & 5.14 \\
\hline 1 & 70 & BJ & $93.97(0.10)$ & $5.91 / 0.12$ & $5.41(0.03)$ \\
\hline & & $\mathrm{S}$ & $91.79(0.30)$ & $3.62 / 4.58$ & $5.30(0.04)$ \\
\hline $\mathrm{h}$ & $\mathrm{n}$ & Theoretical & $95 \%$ & $2.50 \% / 2.50 \%$ & 5.15 \\
\hline 2 & 70 & BJ & $93.99(0.12)$ & $5.85 / 0.16$ & $5.44(0.03)$ \\
\hline & & $\mathrm{S}$ & $92.37(0.28)$ & $3.56 / 4.07$ & $5.30(0.04)$ \\
\hline $\mathrm{h}$ & $\mathrm{n}$ & Theoretical & $95 \%$ & $2.50 \% / 2.50 \%$ & 5.26 \\
\hline 3 & 70 & BJ & $94.31(0.09)$ & $5.64 / 0.05$ & $5.53(0.03)$ \\
\hline & & $\mathrm{S}$ & $94.02(0.18)$ & $3.56 / 2.42$ & $5.43(0.04)$ \\
\hline $\mathrm{h}$ & $\mathrm{n}$ & Theoretical & $95 \%$ & $2.50 \% / 2.50 \%$ & 5.14 \\
\hline 1 & 130 & BJ & $94.33(0.07)$ & $5.67 / 0.00$ & $5.42(0.02)$ \\
\hline & & S & $92.71(0.25)$ & $3.32 / 3.98$ & $5.20(0.03)$ \\
\hline $\mathrm{h}$ & $\mathrm{n}$ & Theoretical & $95 \%$ & $2.50 \% / 2.50 \%$ & 5.15 \\
\hline 2 & 130 & BJ & $94.42(0.07)$ & $5.58 / 0.00$ & $5.43(0.02)$ \\
\hline & & S & $93.85(0.21)$ & $3.29 / 2.87$ & $5.22(0.03)$ \\
\hline $\mathrm{h}$ & $\mathrm{n}$ & Theoretical & $95 \%$ & $2.50 \% / 2.50 \%$ & 5.26 \\
\hline 3 & 130 & BJ & $94.58(0.06)$ & $5.40 / 0.02$ & $5.51(0.02)$ \\
\hline & & S & $94.27(0.17)$ & $3.30 / 2.43$ & $5.31(0.03)$ \\
\hline
\end{tabular}

Table 4: Simulation results for Model 2, with Gaussian residuals.

\begin{tabular}{|c|c|c|c|c|c|}
\hline Lag & Sample size & Method & $C_{M}(\mathrm{se})$ & Cov. (i./s.) & $L_{M}(\mathrm{se})$ \\
\hline $\mathrm{h}$ & $\mathrm{n}$ & Theoretical & $95 \%$ & $2.50 \% / 2.50 \%$ & 0.25 \\
\hline 1 & 80 & BJ & $94.13(0.08)$ & $2.89 / 2.98$ & $0.25(0.00)$ \\
\hline & & $\mathrm{S}$ & $91.55(0.19)$ & $4.15 / 4.31$ & $0.25(0.00)$ \\
\hline $\mathrm{h}$ & $\mathrm{n}$ & Theoretical & $95 \%$ & $2.50 \% / 2.50 \%$ & 0.35 \\
\hline 2 & 80 & BJ & $94.15(0.08)$ & $2.88 / 2.97$ & $0.35(0.00)$ \\
\hline & & $\mathrm{S}$ & $91.98(0.17)$ & $3.96 / 4.06$ & $0.35(0.00)$ \\
\hline $\mathrm{h}$ & $\mathrm{n}$ & Theoretical & $95 \%$ & $2.50 \% / 2.50 \%$ & 0.43 \\
\hline 3 & 80 & BJ & $94.14(0.08)$ & $2.91 / 2.95$ & $0.43(0.00)$ \\
\hline & & $\mathrm{S}$ & $92.05(0.17)$ & $3.93 / 4.01$ & $0.43(0.00)$ \\
\hline $\mathrm{h}$ & $\mathrm{n}$ & Theoretical & $95 \%$ & $2.50 \% / 2.50 \%$ & 0.25 \\
\hline 1 & 160 & BJ & $94.65(0.06)$ & $2.61 / 2.74$ & $0.25(0.00)$ \\
\hline & & $\mathrm{S}$ & $92.80(0.12)$ & $3.61 / 3.58$ & $0.25(0.00)$ \\
\hline $\mathrm{h}$ & $\mathrm{n}$ & Theoretical & $95 \%$ & $2.50 \% / 2.50 \%$ & 0.35 \\
\hline 2 & 160 & BJ & $94.66(0.05)$ & $2.60 / 2.74$ & $0.35(0.00)$ \\
\hline & & S & $92.97(0.11)$ & $3.61 / 3.42$ & $0.35(0.00)$ \\
\hline $\mathrm{h}$ & $\mathrm{n}$ & Theoretical & $95 \%$ & $2.50 \% / 2.50 \%$ & 0.43 \\
\hline 3 & 160 & BJ & $94.59(0.06)$ & $2.63 / 2.78$ & $0.43(0.00)$ \\
\hline & & S & $92.90(0.12)$ & $3.71 / 3.39$ & $0.43(0.00)$ \\
\hline
\end{tabular}


Table 5: Simulation results for Model 2, with exponential residuals.

\begin{tabular}{|c|c|c|c|c|c|}
\hline Lag & Sample size & Method & $C_{M}(\mathrm{se})$ & Cov. (i./s.) & $L_{M}(\mathrm{se})$ \\
\hline $\mathrm{h}$ & $\mathrm{n}$ & Theoretical & $95 \%$ & $2.50 \% / 2.50 \%$ & 0.23 \\
\hline 1 & 80 & BJ & $94.15(0.09)$ & $0.10 / 5.75$ & $0.24(0.00)$ \\
\hline & & $\mathrm{S}$ & $91.84(0.39)$ & $4.38 / 3.77$ & $0.26(0.00)$ \\
\hline $\mathrm{h}$ & $\mathrm{n}$ & Theoretical & $95 \%$ & $2.50 \% / 2.50 \%$ & 0.34 \\
\hline 2 & 80 & BJ & $94.36(0.08)$ & $0.12 / 5.51$ & $0.34(0.00)$ \\
\hline & & $\mathrm{S}$ & $91.86(0.32)$ & $4.11 / 4.03$ & $0.36(0.00)$ \\
\hline $\mathrm{h}$ & $\mathrm{n}$ & Theoretical & $95 \%$ & $2.50 \% / 2.50 \%$ & 0.42 \\
\hline 3 & 80 & BJ & $94.35(0.09)$ & $0.32 / 5.34$ & $0.42(0.00)$ \\
\hline \hline $\mathrm{h}$ & $\mathrm{n}$ & Theoretical & $95 \%$ & $2.50 \% / 2.50 \%$ & 0.23 \\
\hline 1 & 160 & BJ & $94.68(0.05)$ & $0.00 / 5.32$ & $0.25(0.00)$ \\
\hline & & S & $92.37(0.37)$ & $4.31 / 3.32$ & $0.25(0.00)$ \\
\hline $\mathrm{h}$ & $\mathrm{n}$ & Theoretical & $95 \%$ & $2.50 \% / 2.50 \%$ & 0.34 \\
\hline 2 & 160 & BJ & $94.89(0.05)$ & $0.01 / 5.10$ & $0.35(0.00)$ \\
\hline & & S & $92.42(0.28)$ & $4.17 / 3.41$ & $0.35(0.00)$ \\
\hline $\mathrm{h}$ & $\mathrm{n}$ & Theoretical & $95 \%$ & $2.50 \% / 2.50 \%$ & 0.42 \\
\hline 3 & 160 & BJ & $95.04(0.05)$ & $0.08 / 4.88$ & $0.43(0.00)$ \\
\hline & & S & $92.78(0.22)$ & $3.79 / 3.42$ & $0.43(0.00)$ \\
\hline
\end{tabular}

Table 6: Simulation results for Model 2, with -exponential residuals.

\begin{tabular}{|c|c|c|c|c|c|}
\hline Lag & Sample size & Method & $C_{M}(\mathrm{se})$ & Cov. (i./s.) & $L_{M}(\mathrm{se})$ \\
\hline $\mathrm{h}$ & $\mathrm{n}$ & Theoretical & $95 \%$ & $2.50 \% / 2.50 \%$ & 0.23 \\
\hline 1 & 80 & BJ & $94.50(0.07)$ & $5.44 / 0.05$ & $0.25(0.00)$ \\
\hline & & $\mathrm{S}$ & $92.03(0.38)$ & $3.54 / 4.42$ & $0.26(0.00)$ \\
\hline $\mathrm{h}$ & $\mathrm{n}$ & Theoretical & $95 \%$ & $2.50 \% / 2.50 \%$ & 0.34 \\
\hline 2 & 80 & BJ & $94.58(0.08)$ & $5.30 / 0.11$ & $0.35(0.00)$ \\
\hline & & $\mathrm{S}$ & $92.18(0.31)$ & $3.72 / 4.11$ & $0.36(0.00)$ \\
\hline $\mathrm{h}$ & $\mathrm{n}$ & Theoretical & $95 \%$ & $2.50 \% / 2.50 \%$ & 0.42 \\
\hline 3 & 80 & BJ & $94.60(0.08)$ & $5.11 / 0.28$ & $0.43(0.00)$ \\
\hline & & $\mathrm{S}$ & $91.67(0.29)$ & $3.95 / 4.37$ & $0.44(0.00)$ \\
\hline \hline $\mathrm{h}$ & $\mathrm{n}$ & Theoretical & $95 \%$ & $2.50 \% / 2.50 \%$ & 0.23 \\
\hline 1 & 160 & BJ & $94.58(0.05)$ & $5.40 / 0.02$ & $0.25(0.00)$ \\
\hline & & $\mathrm{S}$ & $92.32(0.37)$ & $3.29 / 4.38$ & $0.25(0.00)$ \\
\hline $\mathrm{h}$ & $\mathrm{n}$ & Theoretical & $95 \%$ & $2.50 \% / 2.50 \%$ & 0.34 \\
\hline 2 & 160 & BJ & $94.86(0.05)$ & $5.11 / 0.02$ & $0.35(0.00)$ \\
\hline & & S & $92.57(0.29)$ & $3.36 / 4.07$ & $0.35(0.00)$ \\
\hline $\mathrm{h}$ & $\mathrm{n}$ & Theoretical & $95 \%$ & $2.50 \% / 2.50 \%$ & 0.42 \\
\hline 3 & 160 & BJ & $95.07(0.05)$ & $4.87 / 0.06$ & $0.43(0.00)$ \\
\hline & & S & $92.62(0.25)$ & $3.42 / 3.96$ & $0.43(0.00)$ \\
\hline
\end{tabular}


Table 7: Simulation results for Model 3, with Gaussian residuals.

\begin{tabular}{|c|c|c|c|c|c|}
\hline Lag & Sample size & Method & $C_{M}(\mathrm{se})$ & Cov. (i./s.) & $L_{M}(\mathrm{se})$ \\
\hline $\mathrm{h}$ & $\mathrm{n}$ & Theoretical & $95 \%$ & $2.50 \% / 2.50 \%$ & 42.65 \\
\hline 1 & 120 & $\mathrm{BJ}$ & $94.12(0.08)$ & $2.87 / 3.01$ & $42.28(0.11)$ \\
\hline & & $\mathrm{S}$ & $93.24(0.16)$ & $3.13 / 3.63$ & $44.28(0.15)$ \\
\hline $\mathrm{h}$ & $\mathrm{n}$ & Theoretical & $95 \%$ & $2.50 \% / 2.50 \%$ & 45.25 \\
\hline 2 & 120 & $\mathrm{BJ}$ & $94.16(0.07)$ & $2.83 / 3.01$ & $44.81(0.12)$ \\
\hline & & $\mathrm{S}$ & $93.50(0.14)$ & $3.23 / 3.27$ & $46.92(0.14)$ \\
\hline $\mathrm{h}$ & $\mathrm{n}$ & Theoretical & $95 \%$ & $2.50 \% / 2.50 \%$ & 45.56 \\
\hline 3 & 120 & BJ & $94.26(0.07)$ & $2.78 / 2.97$ & $45.21(0.12)$ \\
\hline & & $\mathrm{S}$ & $93.57(0.14)$ & $3.32 / 3.11$ & $47.33(0.14)$ \\
\hline \hline $\mathrm{h}$ & $\mathrm{n}$ & Theoretical & $95 \%$ & $2.50 \% / 2.50 \%$ & 42.65 \\
\hline 1 & 240 & BJ & $94.72(0.05)$ & $2.59 / 2.68$ & $42.68(0.07)$ \\
\hline & & $\mathrm{S}$ & $93.57(0.11)$ & $3.20 / 3.23$ & $43.50(0.11)$ \\
\hline $\mathrm{h}$ & $\mathrm{n}$ & Theoretical & $95 \%$ & $2.50 \% / 2.50 \%$ & 45.25 \\
\hline 2 & 240 & BJ & $94.66(0.05)$ & $2.61 / 2.73$ & $45.16(0.08)$ \\
\hline & & $\mathrm{S}$ & $93.79(0.10)$ & $3.00 / 3.21$ & $46.03(0.10)$ \\
\hline $\mathrm{h}$ & $\mathrm{n}$ & Theoretical & $95 \%$ & $2.50 \% / 2.50 \%$ & 45.56 \\
\hline 3 & 240 & BJ & $94.76(0.05)$ & $2.56 / 2.68$ & $45.60(0.09)$ \\
\hline & & S & $93.78(0.10)$ & $3.03 / 3.19$ & $46.39(0.10)$ \\
\hline
\end{tabular}

Table 8: Simulation results for Model 3, with exponential residuals.

\begin{tabular}{|c|c|c|c|c|c|}
\hline Lag & Sample size & Method & $C_{M}(\mathrm{se})$ & Cov. (i./s.) & $L_{M}(\mathrm{se})$ \\
\hline $\mathrm{h}$ & $\mathrm{n}$ & Theoretical & $95 \%$ & $2.50 \% / 2.50 \%$ & 39.84 \\
\hline 1 & 120 & $\mathrm{BJ}$ & $94.38(0.06)$ & $0.02 / 5.61$ & $42.01(0.18)$ \\
\hline & & $\mathrm{S}$ & $94.08(0.30)$ & $2.61 / 3.32$ & $44.86(0.25)$ \\
\hline $\mathrm{h}$ & $\mathrm{n}$ & Theoretical & $95 \%$ & $2.50 \% / 2.50 \%$ & 42.98 \\
\hline 2 & 120 & $\mathrm{BJ}$ & $94.54(0.07)$ & $0.07 / 5.39$ & $44.62(0.20)$ \\
\hline & & $\mathrm{S}$ & $93.66(0.26)$ & $3.06 / 3.28$ & $47.11(0.24)$ \\
\hline $\mathrm{h}$ & $\mathrm{n}$ & Theoretical & $95 \%$ & $2.50 \% / 2.50 \%$ & 43.74 \\
\hline 3 & 120 & BJ & $94.53(0.06)$ & $0.04 / 5.44$ & $44.96(0.20)$ \\
\hline & & $\mathrm{S}$ & $93.88(0.26)$ & $2.77 / 3.35$ & $47.48(0.24)$ \\
\hline \hline $\mathrm{h}$ & $\mathrm{n}$ & Theoretical & $95 \%$ & $2.50 \% / 2.50 \%$ & 39.84 \\
\hline 1 & 240 & BJ & $94.60(0.04)$ & $0.00 / 5.40$ & $42.56(0.13)$ \\
\hline & & $\mathrm{S}$ & $94.03(0.27)$ & $2.87 / 3.10$ & $43.47(0.18)$ \\
\hline $\mathrm{h}$ & $\mathrm{n}$ & Theoretical & $95 \%$ & $2.50 \% / 2.50 \%$ & 43.09 \\
\hline 2 & 240 & BJ & $94.83(0.04)$ & $0.00 / 5.17$ & $45.16(0.14)$ \\
\hline & & $\mathrm{S}$ & $93.75(0.25)$ & $3.21 / 3.03$ & $46.05(0.17)$ \\
\hline $\mathrm{h}$ & $\mathrm{n}$ & Theoretical & $95 \%$ & $2.50 \% / 2.50 \%$ & 43.55 \\
\hline 3 & 240 & BJ & $94.82(0.04)$ & $0.00 / 5.18$ & $45.47(0.14)$ \\
\hline & & S & $94.11(0.24)$ & $2.92 / 2.97$ & $46.51(0.17)$ \\
\hline
\end{tabular}


Table 9: Simulation results for Model 3, with -exponential residuals.

\begin{tabular}{|c|c|c|c|c|c|}
\hline Lag & Sample size & Method & $C_{M}(\mathrm{se})$ & Cov. (i./s.) & $L_{M}(\mathrm{se})$ \\
\hline $\mathrm{h}$ & $\mathrm{n}$ & Theoretical & $95 \%$ & $2.50 \% / 2.50 \%$ & 40.07 \\
\hline 1 & 120 & BJ & $94.45(0.06)$ & $5.55 / 0.00$ & $41.93(0.18)$ \\
\hline & & $\mathrm{S}$ & $94.13(0.28)$ & $3.30 / 2.57$ & $44.97(0.25)$ \\
\hline $\mathrm{h}$ & $\mathrm{n}$ & Theoretical & $95 \%$ & $2.50 \% / 2.50 \%$ & 43.37 \\
\hline 2 & 120 & BJ & $94.56(0.06)$ & $5.43 / 0.01$ & $44.38(0.19)$ \\
\hline & & $\mathrm{S}$ & $93.62(0.29)$ & $3.18 / 3.20$ & $47.48(0.24)$ \\
\hline $\mathrm{h}$ & $\mathrm{n}$ & Theoretical & $95 \%$ & $2.50 \% / 2.50 \%$ & 43.94 \\
\hline 3 & 120 & BJ & $94.56(0.08)$ & $5.36 / 0.08$ & $44.83(0.19)$ \\
\hline & & $\mathrm{S}$ & $93.76(0.27)$ & $3.21 / 3.03$ & $47.78(0.24)$ \\
\hline \hline $\mathrm{h}$ & $\mathrm{n}$ & Theoretical & $95 \%$ & $2.50 \% / 2.50 \%$ & 40.07 \\
\hline 1 & 240 & BJ & $94.67(0.04)$ & $5.33 / 0.00$ & $42.43(0.13)$ \\
\hline & & S & $93.67(0.30)$ & $2.97 / 3.36$ & $43.82(0.18)$ \\
\hline $\mathrm{h}$ & $\mathrm{n}$ & Theoretical & $95 \%$ & $2.50 \% / 2.50 \%$ & 43.37 \\
\hline 2 & 240 & BJ & $94.90(0.04)$ & $5.10 / 0.00$ & $45.01(0.14)$ \\
\hline & & S & $93.59(0.27)$ & $2.99 / 3.43$ & $46.17(0.17)$ \\
\hline $\mathrm{h}$ & $\mathrm{n}$ & Theoretical & $95 \%$ & $2.50 \% / 2.50 \%$ & 43.94 \\
\hline 3 & 240 & BJ & $94.89(0.04)$ & $5.11 / 0.00$ & $45.38(0.14)$ \\
\hline & & S & $93.95(0.25)$ & $2.93 / 3.12$ & $46.73(0.18)$ \\
\hline
\end{tabular}

Table 10: Simulation results for Model 4, with Gaussian residuals.

\begin{tabular}{|c|c|c|c|c|c|}
\hline Lag & Sample size & Method & $C_{M}(\mathrm{se})$ & Cov. (i./s.) & $L_{M}(\mathrm{se})$ \\
\hline $\mathrm{h}$ & $\mathrm{n}$ & Theoretical & $95 \%$ & $2.50 \% / 2.50 \%$ & 6.71 \\
\hline 1 & 260 & BJ & $93.61(0.07)$ & $3.09 / 3.29$ & $6.63(0.01)$ \\
\hline & & $\mathrm{S}$ & $93.13(0.18)$ & $3.56 / 3.32$ & $7.28(0.02)$ \\
\hline $\mathrm{h}$ & $\mathrm{n}$ & Theoretical & $95 \%$ & $2.50 \% / 2.50 \%$ & 6.77 \\
\hline 2 & 260 & BJ & $93.70(0.07)$ & $3.11 / 3.19$ & $6.66(0.01)$ \\
\hline & & $\mathrm{S}$ & $93.42(0.17)$ & $3.37 / 3.22$ & $7.36(0.02)$ \\
\hline $\mathrm{h}$ & $\mathrm{n}$ & Theoretical & $95 \%$ & $2.50 \% / 2.50 \%$ & 6.79 \\
\hline 3 & 260 & BJ & $93.69(0.07)$ & $3.09 / 3.22$ & $6.69(0.01)$ \\
\hline & & $\mathrm{S}$ & $93.73(0.14)$ & $3.32 / 2.95$ & $7.44(0.02)$ \\
\hline $\mathrm{h}$ & $\mathrm{n}$ & Theoretical & $95 \%$ & $2.50 \% / 2.50 \%$ & 6.71 \\
\hline 1 & 520 & BJ & $94.51(0.04)$ & $2.64 / 2.85$ & $6.66(0.01)$ \\
\hline & & S & $94.18(0.10)$ & $2.71 / 3.10$ & $7.10(0.01)$ \\
\hline $\mathrm{h}$ & $\mathrm{n}$ & Theoretical & $95 \%$ & $2.50 \% / 2.50 \%$ & 6.77 \\
\hline 2 & 520 & BJ & $94.42(0.05)$ & $2.74 / 2.84$ & $6.69(0.01)$ \\
\hline & & S & $94.10(0.10)$ & $2.83 / 3.06$ & $7.14(0.01)$ \\
\hline $\mathrm{h}$ & $\mathrm{n}$ & Theoretical & $95 \%$ & $2.50 \% / 2.50 \%$ & 6.79 \\
\hline 3 & 520 & BJ & $94.51(0.05)$ & $2.64 / 2.85$ & $6.74(0.01)$ \\
\hline & & S & $94.39(0.09)$ & $2.80 / 2.81$ & $7.20(0.01)$ \\
\hline
\end{tabular}


Table 11: Simulation results for Model 4, with exponential residuals.

\begin{tabular}{|c|c|c|c|c|c|}
\hline Lag & Sample size & Method & $C_{M}(\mathrm{se})$ & Cov. (i./s.) & $L_{M}(\mathrm{se})$ \\
\hline $\mathrm{h}$ & $\mathrm{n}$ & Theoretical & $95 \%$ & $2.50 \% / 2.50 \%$ & 6.26 \\
\hline 1 & 260 & BJ & $94.18(0.07)$ & $0.04 / 5.78$ & $6.59(0.02)$ \\
\hline & & $\mathrm{S}$ & $93.56(0.36)$ & $3.19 / 3.25$ & $7.57(0.03)$ \\
\hline $\mathrm{h}$ & $\mathrm{n}$ & Theoretical & $95 \%$ & $2.50 \% / 2.50 \%$ & 6.32 \\
\hline 2 & 260 & BJ & $94.24(0.07)$ & $0.08 / 5.68$ & $6.63(0.02)$ \\
\hline & & $\mathrm{S}$ & $94.24(0.30)$ & $2.37 / 3.39$ & $7.62(0.03)$ \\
\hline $\mathrm{h}$ & $\mathrm{n}$ & Theoretical & $95 \%$ & $2.50 \% / 2.50 \%$ & 6.42 \\
\hline 3 & 260 & BJ & $94.27(0.07)$ & $0.04 / 5.69$ & $6.66(0.02)$ \\
\hline \hline $\mathrm{h}$ & $\mathrm{n}$ & Theoretical & $95 \%$ & $2.50 \% / 2.50 \%$ & 6.26 \\
\hline 1 & 520 & BJ & $94.58(0.04)$ & $0.00 / 5.42$ & $6.64(0.01)$ \\
\hline & & S & $94.44(0.29)$ & $2.58 / 2.98$ & $7.18(0.02)$ \\
\hline $\mathrm{h}$ & $\mathrm{n}$ & Theoretical & $95 \%$ & $2.50 \% / 2.50 \%$ & 6.32 \\
\hline 2 & 520 & BJ & $94.63(0.04)$ & $0.00 / 5.37$ & $6.67(0.02)$ \\
\hline & & S & $94.59(0.28)$ & $2.46 / 2.95$ & $7.24(0.02)$ \\
\hline $\mathrm{h}$ & $\mathrm{n}$ & Theoretical & $95 \%$ & $2.50 \% / 2.50 \%$ & 6.42 \\
\hline 3 & 520 & BJ & $94.64(0.04)$ & $0.01 / 5.35$ & $6.70(0.02)$ \\
\hline & & S & $94.80(0.24)$ & $2.24 / 2.96$ & $7.29(0.02)$ \\
\hline
\end{tabular}

Table 12: Simulation results for Model 4, with -exponential residuals.

\begin{tabular}{|c|c|c|c|c|c|}
\hline Lag & Sample size & Method & $C_{M}(\mathrm{se})$ & Cov. (i./s.) & $L_{M}(\mathrm{se})$ \\
\hline $\mathrm{h}$ & $\mathrm{n}$ & Theoretical & $95 \%$ & $2.50 \% / 2.50 \%$ & 6.33 \\
\hline 1 & 260 & BJ & $94.28(0.07)$ & $5.62 / 0.10$ & $6.59(0.02)$ \\
\hline & & $\mathrm{S}$ & $93.87(0.35)$ & $3.09 / 3.04$ & $7.62(0.03)$ \\
\hline $\mathrm{h}$ & $\mathrm{n}$ & Theoretical & $95 \%$ & $2.50 \% / 2.50 \%$ & 6.43 \\
\hline 2 & 260 & BJ & $94.23(0.08)$ & $5.60 / 0.17$ & $6.62(0.02)$ \\
\hline & & $\mathrm{S}$ & $93.87(0.34)$ & $3.08 / 3.05$ & $7.64(0.03)$ \\
\hline $\mathrm{h}$ & $\mathrm{n}$ & Theoretical & $95 \%$ & $2.50 \% / 2.50 \%$ & 6.48 \\
\hline 3 & 260 & BJ & $94.26(0.07)$ & $5.66 / 0.08$ & $6.65(0.02)$ \\
\hline & & $\mathrm{S}$ & $94.35(0.29)$ & $3.11 / 2.55$ & $7.73(0.03)$ \\
\hline $\mathrm{h}$ & $\mathrm{n}$ & Theoretical & $95 \%$ & $2.50 \% / 2.50 \%$ & 6.33 \\
\hline 1 & 520 & BJ & $94.61(0.04)$ & $5.37 / 0.02$ & $6.65(0.02)$ \\
\hline & & S & $94.32(0.28)$ & $2.83 / 2.86$ & $7.24(0.02)$ \\
\hline $\mathrm{h}$ & $\mathrm{n}$ & Theoretical & $95 \%$ & $2.50 \% / 2.50 \%$ & 6.43 \\
\hline 2 & 520 & BJ & $94.66(0.04)$ & $5.34 / 0.00$ & $6.68(0.02)$ \\
\hline & & S & $94.58(0.29)$ & $2.78 / 2.64$ & $7.29(0.02)$ \\
\hline $\mathrm{h}$ & $\mathrm{n}$ & Theoretical & $95 \%$ & $2.50 \% / 2.50 \%$ & 6.48 \\
\hline 3 & 520 & BJ & $94.64(0.04)$ & $5.36 / 0.00$ & $6.71(0.02)$ \\
\hline & & S & $94.75(0.28)$ & $2.84 / 2.41$ & $7.32(0.02)$ \\
\hline
\end{tabular}

